\title{
Multi-domain overarching control of flexi-grid networks with GMPLS as Inter-SDN controller communication
}

\author{
Ramon Casellas (1), Ricardo Martínez (1), Raül Muñoz (1), Ricard Vilalta (1), Lei Liu (2) \\ (1) CTTC, Av. Carl Friedrich Gauss 77 - PMT Edifici B4-08860 Castelldefels, Barcelona, Spain \\ (2) University of California, Davis, One Shields Ave, Davis, CA 95616, USA \\ ramon.casellas@cttc.es
}

Abstract: We report the experimental assessment of a hybrid control plane for multi-domain, heterogeneous networks based on abstraction and hierarchical TE. A mesh of SDN controllers use GMPLS protocols as East/West interfaces, forming an abstracted topology.

OCIS codes: (060.4250) Networks; (060.4251) Networks, assignment and routing algorithms

\section{Introduction, Motivation, Identified Use Cases and Requirements}

Simple SDN control deployments are scoped to a single domain, where a (logically) centralized controller has full topology visibility of the network. Even if the network is segmented, a common deployment still considers a single controller. However, new use cases and scenarios call for more robust and scalable solutions addressing multidomain scenarios, such as heterogeneous control plane interworking (SDN/OpenFlow [1] and GMPLS [2]) or networks segmented in vendor islands, potentially involving multiple controllers. Additionally, Traffic Engineering (TE) information is contained within each domain for scaling and confidentiality, but end-to-end services may require a constrained TE path, so it is efficient to expose a limited amount of TE information. In [3], we proposed a multiple controller model for overarching control and orchestration based on a hierarchy. In this work, we consider a hybrid solution, based on a combination of centralized and distributed elements, where a mesh of controllers collaborate running a dedicated GMPLS control plane instance [4], as opposed to authors in [5] which develop a dedicated inter domain protocol based on PCEP. We consider the concept of TE reachability as defined in [6], so that a client can determine whether they can establish a multi-domain TE path. Key to this is TE network abstraction, which is the synthesizing of reported TE attribute information for each domain and inter-domain links (abstract topology). This representation does not include all possible connectivity options, but instead provides a connectivity matrix based on attributes as reported from within each domain. The abstraction makes use of available TE information, but is subject to network policy and management. The proposed architecture addresses several use cases such as multi-domain over-arching control, migration scenarios, or support for GMPLS UNI for an SDN controller. It combines the benefits of the GMPLS/ASON routing (i.e., robustness, scalability, stability) while locally enabling network operators and SDN in their scoped and controlled domains (control and data plane separation, logically centralized controllers, open interfaces and modular software designs).

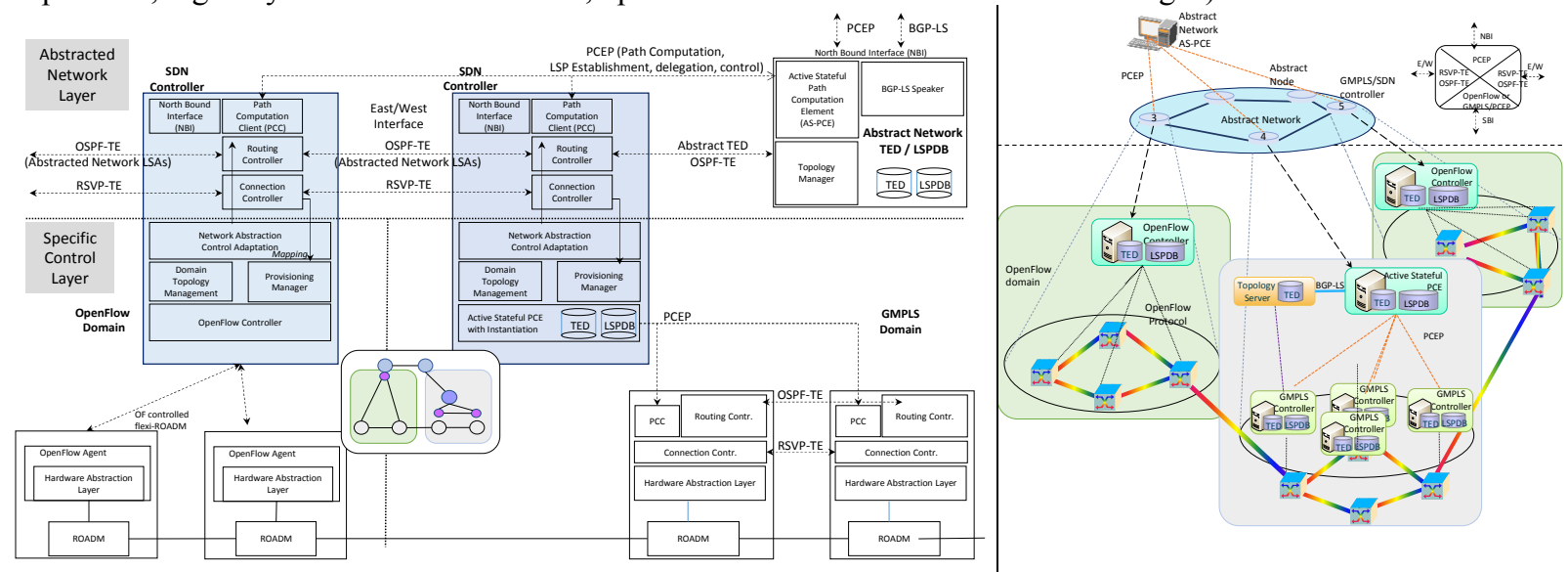

Fig. 1 Hybrid control plane architecture based on a mesh of SDN controllers forming an abstracted network layer

2. Proposed Architecture and Control Procedures

We design, implement, deploy and validate a control plane architecture for multi-domain flexi-grid optical network (Fig.1). It is based on a mesh of SDN controllers that implement GMPLS routing and signaling as their East/West interfaces (i.e., GMPLS as inter-SDN controller communication), and either OpenFlow or GMPLS as intra-domain control, a trade-off between flexibility, scalability and availability. Cooperating SDN controllers are responsible for the abstraction of their domain (in terms of announcing an underlying abstract topology) and for the adaptation of 
the control procedures, which remains technology specific. This effectively introduces a hierarchy with an abstracted network layer and topology. Note the recursive property of the architecture, where the abstract network can be in turn placed behind a SDN controller or active stateful PCE (AS-PCE) and be part of a wider orchestration and over-arching control layer (i.e., to jointly provision IT and network resources).

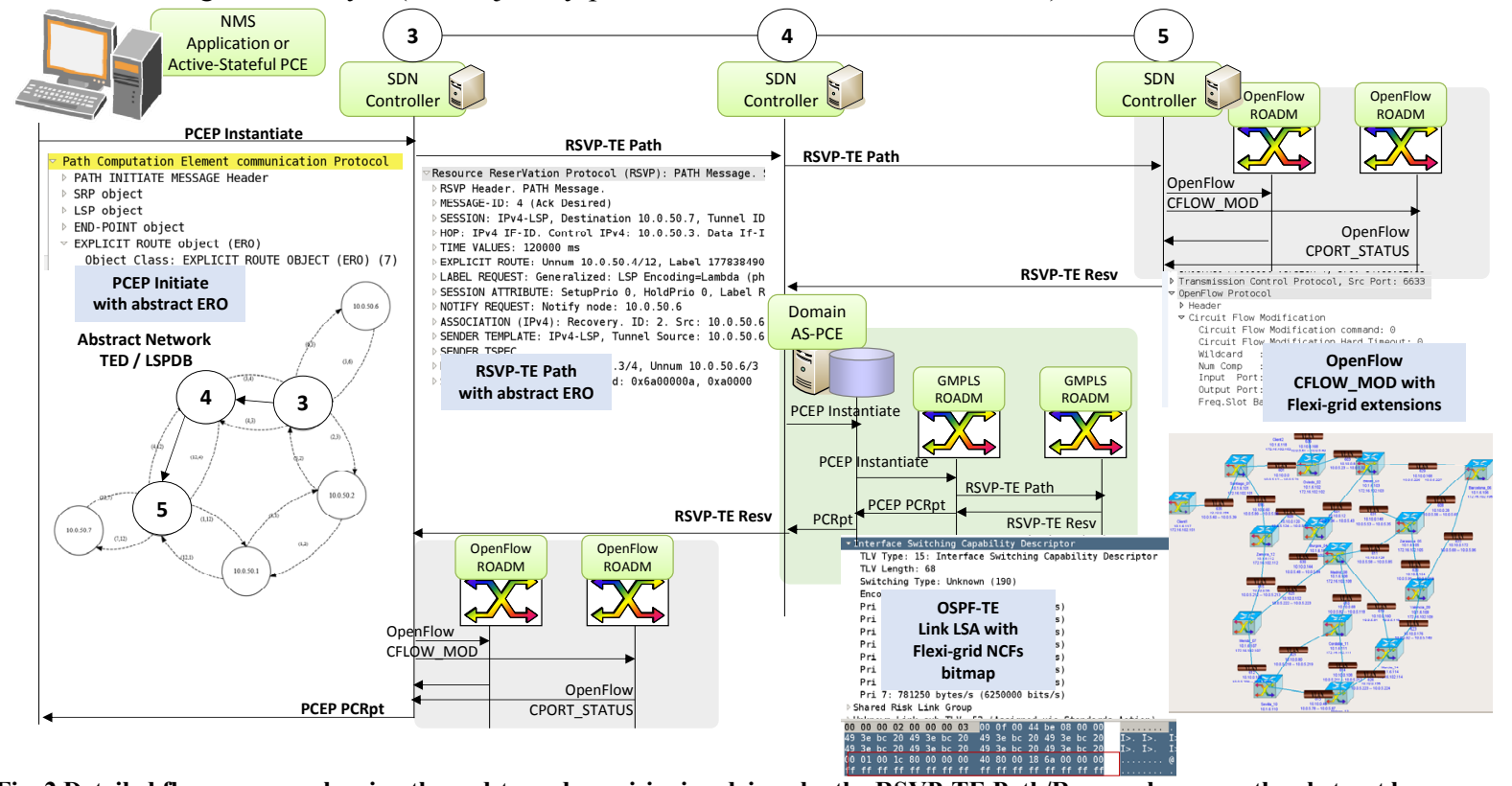

Fig. 2 Detailed flow message showing the end-to-end provisioning driven by the RSVP-TE Path/Resv exchange on the abstract layer We consider a domain as a set of ROADMs under the control of a technology specific control plane, assuming a deployment model of a single SDN controller per domain with full topology visibility. The SDN controller is extended with GMPLS protocols as its East/West interfaces. Such GMPLS-enabled SDN controllers (GSCs) maintain a domain abstract topology map and execute a separate GMPLS instance in which routing and signaling adjacencies are congruent to the domain connectivity. Each GSC disseminates the abstraction of its domain by means of dedicated OSPF-TE Link State Advertisements (LSAs), and end-to-end provisioning (Fig.2) is triggered by the AS-PCE in the abstract network, e.g., by sending a PCE Communication Protocol (PCEP) initiate message to the ingress abstract node, and relying on distributed signaling over the Data Communication Network (DCN) formed by the controller mesh. Each GSC processes RSVP-TE signaling messages, mapping resource reservations to actual segment connectivity requests, which are, in turn delegated to the underlying, specific technology (e.g., a cross-connect at an abstract nodes between input and output ports induces a segment connection in the corresponding domain). Concretely, a receiving GSC processes the Path and Resv messages and identifies the involved abstract TE links, from the Path RSVP_HOP and ERO objects, mapping such abstract link identifiers to its domain client port addresses. The sender descriptor traffic parameters (SENDER_TSPEC) object conveys the requested frequency slot width and, if available, SUGGESTED_LABEL objects may specify the upstream domain preference, whereas the Resv GENERALIZED_LABEL contains the downstream allocated frequency slot. The underlying segment is established either using GMPLS/PCE control plane (domain 4 in the Figure 2) or OpenFlow (domains 3 and 5), since the GSC south bound interface (SBI) is technology specific.

\section{Experimental Assessment and Proof-of-Concept with Heterogeneous Control Plane Domains}

We have deployed a control plane testbed with emulated optical hardware (Fig.3) configured as a multi-domain network with 5 domains. Each domain is controlled by a GSC that performs single TE node network abstraction, using OSPF-TE LSAs to disseminate node identifiers (domain X has 32 bit address 10.0.50.X) and link TE attributes. Additional nodes representing GMPLS clients/endpoints -6 and 7 - have been added, resulting in the abstract topology (Fig. 4). Domains 3 and 5 are optical OpenFlow islands (4 nodes each) [7] so the GSC directly programs cross-connects (CFLOW_MOD and CPORT_STATUS messages). Domains 4, 1, 2 are GMPLS with ASPCE to instantiate LSPs. Domain $\overline{4}$, in particular, has 14 nodes representing a Spanish-wide optical mesh network. All optical links have 128 nominal central frequencies. Connections are set up from Endpoint A (represented in the abstract network as node 10.0.50.6 and corresponding to OF datapathid 00-05 in domain 3) to B, for a frequency slot of width $\mathrm{m}=1$. We focus on the feasibility of the approach, measuring setup delay as seen by an application, and its decomposition (Fig. 5). Note that only control plane latencies are considered, without hardware delays. 


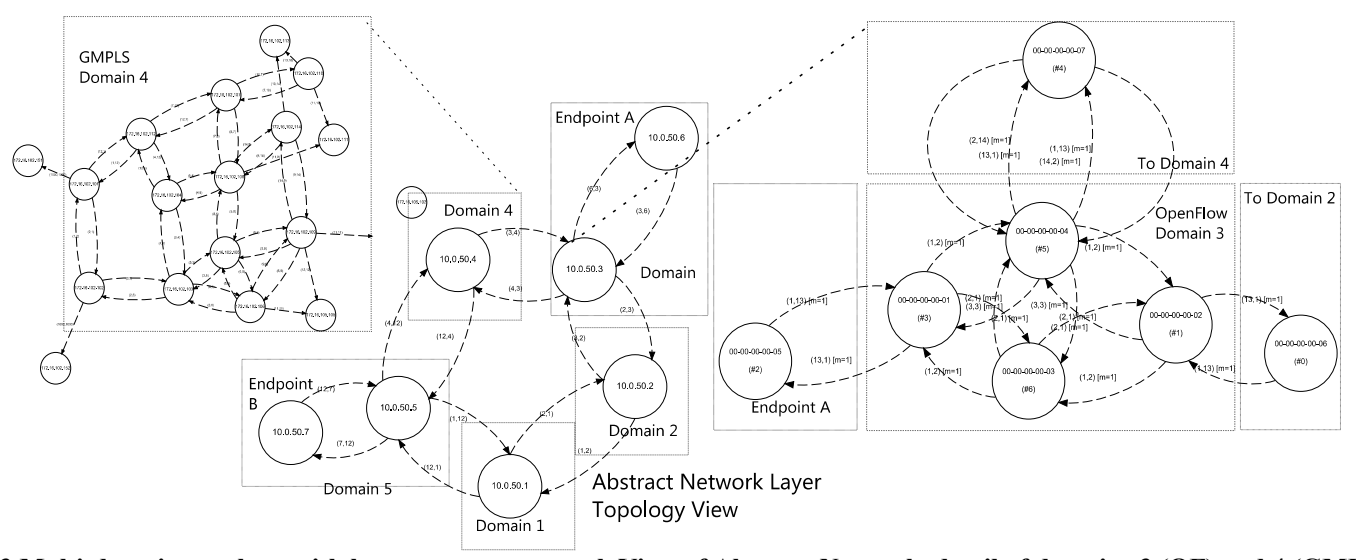

Fig. 3 Multi-domain topology with heterogeneous control. View of Abstract Network, detail of domains 3 (OF) and 4 (GMPLS) Fig. 4 shows segment provisioning in D3 and D4 message exchange (e.g in Dom4 SGC is approximately 109 ms).

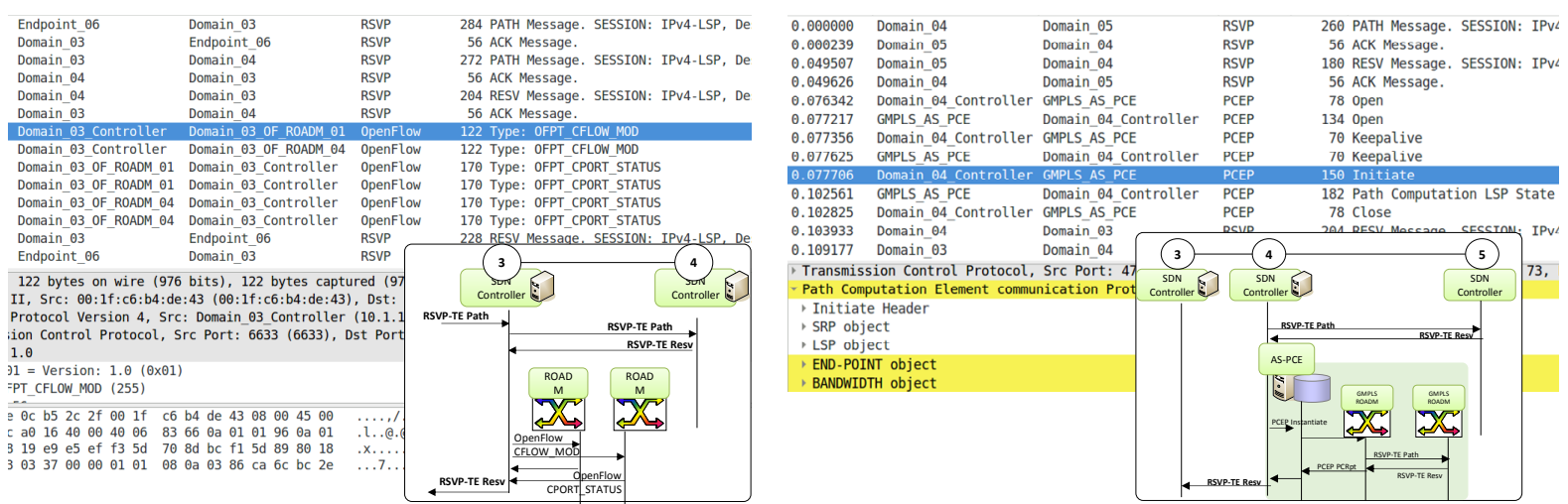

Fig. 4 Wireshark of segment provisioning in Domains 3 (OpenFlow) and 4 (GMPLS) upon reception of Resv from downstream To stress the system, we have requested 750 connections with deterministic arrival and departure of $1 \mathrm{~s}$, using the AS-PCE in the abstract layer NBI. Fig 5 shows the provisioning time histogram and CDF (avg. $\sim 180 \mathrm{~ms}$ ). Control plane overhead is low assuming dedicated control plane links, with message sizes of up $\sim 300$ bytes. The considered scenario validates the approach which scales in the number of domains due to abstraction and distributed nature of GMPLS while assuming that each control domain is reasonably sized (up to tens of nodes).

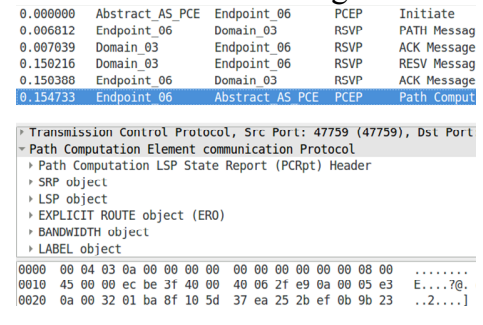

\begin{tabular}{|l|l|}
\hline Control Plane Latency & $\mathrm{ms}$ \\
\hline $\begin{array}{l}\text { Setup delay seen by NMS/App to AS-PCE in } \\
\text { Abstract Network }\end{array}$ & 180 \\
\hline Setup delay seen by ingress abstract node & 155 \\
\hline Segment delay Dom. 4 SDN Controller & 109 \\
\hline $\begin{array}{l}\text { Segm establishment in Dom3 \& 5 (symm. } \\
\text { topology domains) OFP parallel. and ack. }\end{array}$ & 12 \\
\hline Segm setup AS-PCE in Dom4 GMPLS & 30 \\
\hline Segm setup Dom 4 GMPLS & 16 \\
\hline
\end{tabular}

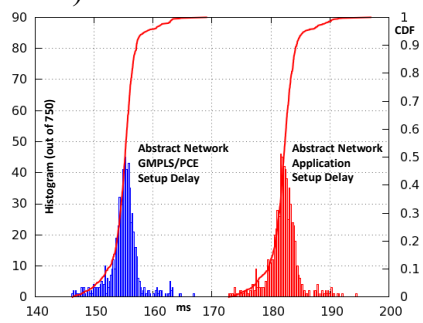

Fig. 5 Wireshark from the abstract network (I) Decomposition of the delay end to end (c) Setup delay histogram and CDF (r)

\section{Conclusions}

We have proposed and implemented an architecture for over-arching control multi-domain optical networks with heterogeneous (OF/GMPLS) control planes based on network abstraction and on a mesh of SDN controllers using GMPLS as East/West interfaces, for scalability and robustness. Experimental validation assesses the approach.

\section{Acknowledgements}

Spanish MINECO FARO (TEC2012-38119), FP7 proj IDEALIST (317999) \& EU-Japan FP7 STRAUSS (608528).

\section{References}

[1] ONF, "OpenFlow Switch Specification, version 1.4 (Wire protocol 0x5)," Open Networking Foundation, October 2013.

[2] E. Mannie Ed., "Generalized Multi-Protocol Label Switching (GMPLS) Architecture,” IETF RFC 3945, October 2004.

[3] R. Casellas, et al., "SDN based Provisioning Orchestration of OpenFlow/GMPLS Flexi-grid Networks with a Stateful H-PCE," OFC2014

[4] R. Muñoz, et al. "Dynamic and Adaptive Control Plane Solutions for Flexi-Grid Optical Networks Based on Stateful PCE" JLT v32 n16 2014

[5] W. Liu, et al., "A distributed stateful PCE Architecture for providing end-to-end connections in multi-domain multi-vendor", ECOC2014

[6] A. Farrel, "Problem Statement and Architecture for Information Exchange between Interconnected Traffic Engineered Networks", IETF draft

[7] R. Casellas, et al. "Control and management of flexi-grid optical networks with integrated stateful PCE/OpenFlow control” JOCN v5n10 2013 